Copyright $@ 2010$ Institute of Electrical and electronics Engineers, Inc.

All Rights reserved.

Personal use of this material, including one hard copy reproduction, is permitted.

Permission to reprint, republish and/or distribute this material in whole or in part for any other purposes must be obtained from the IEEE.

For information on obtaining permission, send an e-mail message to stds-igr@ieee.org.

By choosing to view this document, you agree to all provisions of the copyright laws protecting it.

Individual documents posted on this site may carry slightly different copyright restrictions.

For specific document information, check the copyright notice at the beginning of each document. 


\section{Determining supply chain flexibility using statistics and neural networks: A comparative study}

\author{
Ananda Jeeva \\ Faculty of Arts, Business, Informatics and \\ Education \\ Central Queensland University \\ Australia \\ a.jeeva@cqu.edu.au
}

\author{
William Guo \\ Faculty of Arts, Business, Informatics and \\ Education \\ Central Queensland University \\ Australia \\ w.guo@cqu.edu.au
}

\begin{abstract}
The purpose of this paper is to examine the application of neural networks as a flexibility and performance measure in supplier-manufacturer activities. The dimensions of information exchange, supplier integration, product delivery, logistics, and organisational structure are used as determinants factors affecting this supply chain flexibility. The data set was collected from more than 200 Australian manufacturing firms evaluating their suppliers. Our study shows that neural networks can accurately determine a supplier's flexibility with an error within $1 \%$, which is more accurate than the conventional multivariate regression can.
\end{abstract}

Keywords: neural network, supply chain, flexibility, multivariate regression

\section{INTRODUCTION}

The current economic climate pushes businesses to be more competitive and thus leads to re-evaluating their supply chain strategies. This competitiveness requires organisations to be more effective, efficient and productive with little margin for error. It is also pushing companies to work more strategically with their supply partners. Hence strategic supply partnership is becoming more critical and will determine the sustainability of companies in the future (Lambert \& Cooper 2000). Competitiveness takes many forms, but this paper focuses on the parts of the supply chain activities that inter-relate with suppliers in their operational aspects.

The supply chain operational aspects considered here are between manufacturers and their suppliers. Although there are many activities in these functions, specific activities are selected. The selected activities are related to the interactions of the responding manufacturing firm with their suppliers. These activities are information exchange, the level of supplier integration, product and delivery variations, logistics, and the manufacturing organisational capability. These activities are selected as they represent some of the principal components of supply chain literature as proposed by Croom, Romano and Giannakis (2000).

These selected activities are hypothesised to have the highest ability and agility to be flexible. This flexibility is related to changes in the external and internal environment. As the factors in the external environment change, these supply chain activities are also expected to change accordingly to maintain the same level of efficiency and effectiveness. Flexibility and agility has been promulgated as strategic responses to the stochastic economy (Swafford et al. 2006, Duclos et al. 2003, Beach et al. 2000). This paper focuses on a few determinate factors of supply chain flexibility to predict performance by flexibility measures. We describe two systems that use statistical and artificial neural network techniques respectively for determining the flexibility of suppliers based on their selected elements. The data set used in this study was collected from 241 manufacturers commenting about the flexibility based on the five attributes of supplier-manufacturer activities. A comparison is also made between these two approaches.

\section{ELEMENTS DETERMINING SUPPLY CHAIN FLEXIBILITY}

The information exchange (IE) investigates the elements of range of information from suppliers, such as sufficiency of information, reliability of information, timeliness, accuracy, easy of sending, amount of human intervention required, and connectivity and compatibility of information systems.

The supplier integration (SI) activity takes into consideration of the elements of suppliers' inventory levels, such as delivery schedule changes, service level standard, costs, ease and time to switch suppliers. The supplier integration activities represent the amount of suppliers' ability to integrate into the manufacturing process. 
Product and delivery (PD) elements include the suppliers' ability to design and deliver new components, to modify the product mix, to implement product design, and to modify components variability. This element relates the suppliers' capability and capacity to adapt to changing final consumer demands.

Supplier logistics (SL) elements are the physical movement and handling of the procured products. They include the modification of routes, transportation, performance and product variability, and materials handling in terms of product attributes.

Organisational strategy (OS) indicates how well the internal structure of the organisation can adapt to changing external environment in terms of the supply chain functions. They include contingency supply strategy, cost of implementation, and responsive actions.

Manufacturing organisations' suppliers that have the most flexibility in the above mentioned activities and elements will be able to respond to their own internal stochastic environment as well as their suppliers' stochastic environment. This flexibility must be built into all the activities and elements discussed above. In order to measure this flexibility, the scale development was adopted from the elements of flexibility as proposed by Koste and Malhotra (1999). These elements of flexibility were developed in a manufacturing environment, but it is process based; hence is applicable in a supply chain environment, which is also process based.

\section{Multivariate REGRESSION ANALYSIS}

Although it is well known that the higher the five selected factors, the higher the flexibility of a supplier, the correlation between the flexibility and five selected factors is still an unknown function. This is because the quantification of the five factors is based on human judgment that varies from person to person; hence correspondingly the flexibility is somehow an estimation based on the five factors.

Traditional model for measuring and analysing such problem is based on multivariate regression, which has been well explained in many textbooks (Hair et al. 2009, Ho 2006, Pallant 2007). We apply this analysis to the 241 entries mapped between the five factors as inputs and the flexibility as output (Flex), which defines a multivariate regression shown below.
Flex $=0.0643-0.8593 I E+0.6009 S I-0.9827 P D-$ $0.8693 S L-0.9538 O S$.

This regression is significant with a coefficient of 0.9852 and a maximum error of just about $10 \%$. The accuracy of determining the flexibility of a supplier using this regression will be discussed later incorporating the results from the neural network simulation.

\section{Neural network SIMULATION}

Neural networks have been widely used in investment prediction and financial analysis (Yang, Li \& Xu 2001, Kryzanowski et al. 1993, Brockett et al. 2006). This paper attempts to use it in a supply chain management environment. The use of neural networks offers several advantages over traditional statistical methods. There are no restrictive assumptions as imposed by traditional methodologies. Neural networks use all available information while incorporating new information through a learning process. It updates the old output and learns from its experience. Therefore it is flexible and adaptable and can be used in a changing environment like stochastic economy. Supply chain management activities need to be flexible to respond to external environmental uncertainties.

The core of a neural network is actually an adaptive mathematical model that is capable of approximating any arbitrary unknown function constrained by training datasets. It has been proven that a three-layer multilayer perceptron (MLP) neural network can approximate any continuous function mapped from one finite-dimensional space to another by adjusting the number of nodes in the hidden layer (Hornik et al., 1989). Numerous cases of three-layer MLP applications have been successful in different fields (e.g., Zhang et al., 2003; Gaudart et al., 2004; Yan et al., 2006; Guo et al., 2009). Figure 1 illustrates the structure of a three-layer MLP with one hidden layer of $L$ nodes, a $p$-dimensional input vector $\boldsymbol{X}$ and a $q$-dimensional output vector $\boldsymbol{Y}$. The relationship between the input and output components for this MLP can be generally expressed as

$y_{k}=\varphi\left(\sum_{j=1}^{L} v_{k j} \psi\left(\sum u_{j i} x_{i}\right)\right)$

where $\varphi$ and $\psi$ are the transfer functions; $u_{j i}$ denotes the input-to-hidden layer weights at the hidden neuron $j$; and $v_{k j}$ is the hidden-to-output layer weights at the output unit $k$. 


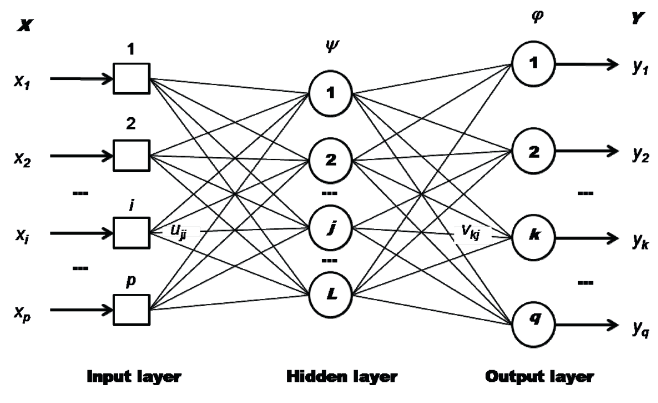

Fig. 1. Three layer multilayer perceptron (MLP) network

The neural network process involves two phases training the network with known datasets and testing the trained network using different known datasets for model generalisation. This is different from statistical approaches, in which the same data can be used for both model generation and evaluation.

In this study, the MLP model is constructed and trained using neural network tools in MATLAB $\mathbb{R}$ (Demuth \& Beale, 2004; Demuth et al., 2007). The Levenberg-Marquardt algorithm (1963) is chosen to train the selected MLPs because this algorithm has been reported to be the fastest method for training moderate-sized feedforward neural networks (Hagan \& Menhaj, 1994; Hagan et al., 1996).

The 241 entries are compiled into two sets. The first set contains $207(86 \%)$ entries for training and the second set includes 34 (14\%) entries for testing. The log-sigmoid-linear combination is chosen as the transfer functions for our MLPs. The running of a number of nodes in the hidden layer shows that a 25-node hidden layer is good enough to achieve satisfactory training (Fig. 2).

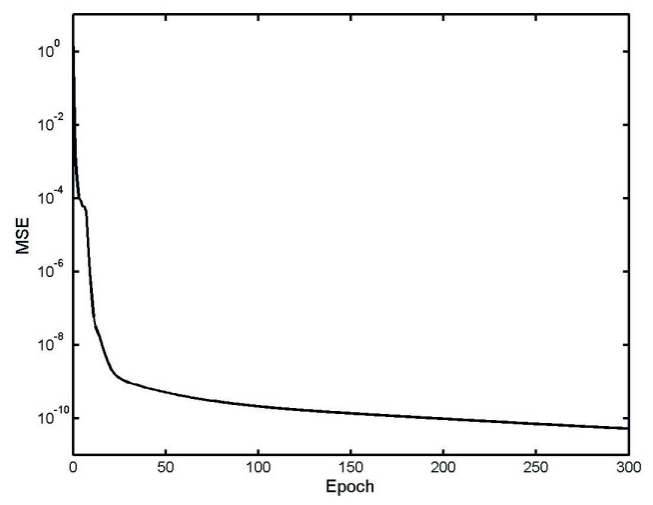

Fig. 2. MLP training pattern

The test results for this 25-neuron MLP are tabulated in Table 1, along with the results for the regression model using the same test set. Both models show good quality for forecasting, but the MLP model is more accurate than the regression model, which can be clearly seen in Fig. 3 .

Table 1. Testing result of MLP and regression models

\begin{tabular}{llllll}
\hline & $\begin{array}{l}\text { Test } \\
\text { size }\end{array}$ & Correlation & $\begin{array}{l}\text { MAE } \\
(\%)\end{array}$ & $\begin{array}{l}\text { SD } \\
(\%)\end{array}$ & $\begin{array}{l}\text { MAX } \\
(\%)\end{array}$ \\
\hline MLP & 34 & 0.9999 & 0.15 & 0.24 & 0.88 \\
\hline Regression & 34 & 0.9829 & 3.12 & 2.53 & 9.43 \\
\hline
\end{tabular}

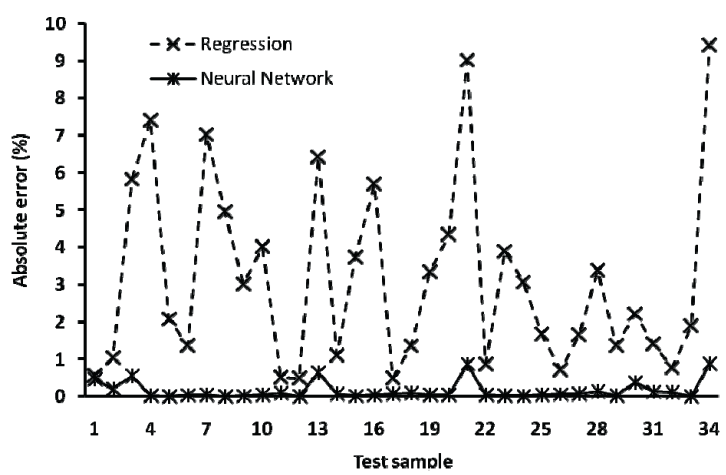

Fig. 3. Plots of targets and simulated outcomes using regression and MLP models

\section{DISCUSSION AND CONCLUSION}

Although the multivariate regression defined in Relation (1) gives an analytical expression that is easier to understand, its performance for predicting a supplier's flexibility through its five attributes may result in an average error of $3 \%$ with a maximum error up to $10 \%$. This is still useful if the manufacturing scale is relatively small. However, if the scale of manufacturing is large, an error of $1 \%$ would mean a loss of millions of dollar in revenue.

The MLP model predicts the flexibility of a supplier with an MAE of less than $0.2 \%$ and a maximum error within $1 \%$. The contrast of these two approaches is clearly illustrated in Fig. 3. Therefore, the neural network model outperforms the statistical model in quantitative simulation. This MLP model creates a dynamic discrete system that is able to approximate the nonlinear relation existing internally among the known entries. It is discrete because by feeding a new entry that falls in the range of the known domain to this neural system, it returns the closest approximation to the most relevant known entries in the domain. It is dynamic because when some new entries are added to the training data, this neural system is able to retrain the model in order to absorb these new entries into its coverage. This either enlarges the known applicable domain if the additional training 
data are beyond the previous range, or refines the local approximating mechanism if the additional training data are within the known domain.

In conclusion, the three-layered MLP is able to predict the supplier's flexibility with respect to its five attributes with a higher accuracy, compared with the multivariate regression analysis. However, multivariate regression is still useful for manufacturers with lesser number of suppliers and can give an understanding on the importance of individual attributes for the flexibility assessment through its analytical expression. This cannot be achieved by neural networks working like a blackbox translator.

\section{REFERENCES}

1. D. M. Lambert and M. C. Cooper, "Issues in Supply Chain Management", Industrial Marketing Management, vol. 29, 2000, pp. 65-83.

2. S. Croom, P. Romano and M. Giannakis, "Supply chain management: An analytical framework for critical review", European Journal of Purchasing and Supply Management, vol. 6, 2000, pp. 67-83.

3. P. M. Swafford, S. Ghosh and N. Murthy, "The antecedents of supply chain agility of a firm: scale development and model testing", Journal of Operations Management, vol. 24, 2006, pp. 170-188.

4. L. K. Duclos, R. J. Vokurka and R.R. Lummus, "A concept model of supply chain flexibility", Industrial Management \& Data Systems, vol. 103, No. 6, 2003, pp. 446-456.

5. R. Beach, A. P. Muhlemann, D. H. R. Price, A. Paterson and J. A. Sharp, "Manufacturing operations and strategic flexibility: survey and cases", International Journal of Operations \& Production Management, vol. 20, 2000, pp. 7-30.

6. L. L. Koste and M. K. Malhotra, "A theoretical framework for analyzing the dimensions of manufacturing flexibility", Journal of Operations Management, vol. 18, 1999, pp. 75 93.
7. J. F. Hair, W. C. Black, B. J. Babin, R. E. Anderson and R. L. Tatham. "Multivariate Data Analysis", 6 ${ }^{\text {th }}$ Edition, Prentic Hall, 2005.

8. R. Ho, "Handbook of Univariate and Multivariate Data Analysis and Interpretation with SPSS", $1^{\text {st }}$ Edition, Chapman \& Hall, 2006

9. J. Pallant, "SPSS Survival Manual: A Step by Step Guide to Data Analysis Using SPSS for Windows", $3{ }^{\text {rd }}$ Edition, Open University Press, 2007.

10. B. Yang, L, X, Li and J. Xu, "An early warning system for load risk assessment using artificial neural networks", Knowledge Based Systems, vol. 14, 2001, pp. 303-306.

11. L. Kryzanowski, M. Galler and D. W. Wright, "Using artificial neural networks to pick stocks", Financial Analysts Journal, vol. 49, No. 4, 1993, pp. 21.

12. P. L. Brockett, L. L. Golden, J. Jang and C. Yang, "A comparison of neural network, statistical methods, and variable choice for life insurers' financial distress prediction, Journal of Risk and Insurance", vol. 73, No. 3, 2006, pp. 397-419.

13. K. Hornik, M. Stinchcomb and H. White, "Multilayer feedforward networks are universal approximators", Neural Networks, vol. 2, 1989, pp. 359-366.

14. G. P. Zhang, M. Keil, A. Rai and J. Mann, "Predicting information technology project escalation: A neural network approach", European Journal of Operational Research, vol. 146, 2003, pp. 115-129.

15. J. Gaudart, B. Giusiano and L. Huiart, "Comparison of the performance of multi-layer perception and linear regression for epidemiological data", Computational Statistics and Data Analysis, vol. 44, 2004, pp 547-570.

16. H. Yan, Y. Jiang, J. Zheng, C. Peng and Q. Li, "A multilayer perceptron based medical decision support system for heart disease diagnosis", Expert Systems with Applications, vol. 30, 2006, pp. 272-281.

17. W. Guo, M. Li, G. Whymark and Z. Li, "Mutual complement between statistical and neural network approaches for rock magnetism data analysis", Expert Systems with Applications, vol. 36, 2009, pp. 9678-9682.

18. H. Demuth and M. Beale, "Neural network toolbox for use with Matlab", Natick, MA: The MathWorks, 2004.

19. H. Demuth, M. Beale and M. Hagan, "Neural network toolbox 5", Natick, MA: The MathWorks, 2007.

20. M. T. Hagan and M. Menhaj, "Training feedforward networks with the Marquardt algorithm", IEEE Transactions on Neural Networks, vol. 5,1994, pp. 989993.

21. M. T. Hagan, H. B. Demuth and M. H. Beale, "Neural network design”, Boston, PWS Publishing, 1996. 